\title{
Targeted Therapies in Triple-Negative Breast Cancer
}

\author{
Frederik Marmé $^{a, b} \quad$ Andreas Schneeweiss ${ }^{a, b}$ \\ a Universitäts-Frauenklinik, University of Heidelberg, Germany; \\ ${ }^{b}$ National Centre for Tumour Diseases, University of Heidelberg, Germany
}

\section{Keywords}

Triple-negative breast cancer - Subtypes .

PARP inhibitors - Immune-checkpoint inhibitors .

Targeted therapy · Bevacizumab

\section{Summary}

Triple-negative breast cancer (TNBC) is a heterogeneous disease comprised of several biologically distinct subtypes. However, treatment is currently mainly relying on chemotherapy as there are no targeted therapies specifically approved for TNBC. Despite initial responses to chemotherapy, resistance frequently and rapidly develops and metastatic TNBC has a poor prognosis. New targeted approaches are, therefore, urgently needed. Currently, bevacizumab, a monoclonal anti-vascular endothelial growth factor (VEGF)-A antibody, is the only targeted agent with an approval for the therapy of metastatic breast cancer, but does not provide a specific benefit in the TNBC subtype. This review discusses the current clinical developments in targeted approaches for TNBC, including anti-angiogenic therapies, epidermal growth factor receptor (EGFR)-targeted therapies, poly(ADP-ribose) polymerase (PARP) inhibitors and platinum salts, as well as novel strategies using immunecheckpoint inhibitors, which have recently demonstrated first promising results. Strategies focusing on specific subtypes of TNBC like anti-androgenic therapies for the luminal androgen receptor subtype (LAR) and others are also discussed.

\section{Introduction}

Triple-negative breast cancer (TNBC) constitutes a heterogeneous group of diseases grouped together because of a shared phenotype, which are merely defined by the lack of expression of the oestrogen receptor (ER), progesterone receptor (PgR) and human epidermal growth factor receptor 2 (HER2). Thus, in contrast to other subtypes, TNBC is not defined by shared phenotypic features but only by their absence.

Disease dynamics, risk of recurrence and patterns of metastasis in TNBC differ from other subtypes. Despite a good response to chemotherapy in primary breast cancer (PBC), TNBCs are associated with a 4-fold increased risk of distant metastases and a significantly shorter overall survival [1-6]. The peak of recurrence risk can be observed early on, within $2-3$ years after initial diagnosis, reflecting a more aggressive course of disease $[3,7,8]$.

A good response to neoadjuvant chemotherapy as defined by a pathological complete remission (pCR) is strongly correlated with very favourable prognosis $[9,10]$. Thus, the unfavourable prognosis of TNBCs is predominantly determined by patients who respond poorly to (neo)adjuvant chemotherapy. Patients not achieving a pCR (about $60 \%$ of TNBC patients) have a 5 -year disease-free survival (DFS) of less than $50 \%$ [10]. In order to improve prognosis for those patients not responding to chemotherapy, novel targeted approaches are urgently needed.

Patients with TNBC develop pulmonary, hepatic and cerebral metastases more frequently and bone, lymphatic or soft tissue metastases less frequently than luminal subtypes [11, 12].

However, as mentioned above, TNBC represents a heterogeneous group and there are some histologically defined subtypes, such as classical medullary, apocrine and adenoid-cystic carcinomas, that are typically triple-negative but have a favourable prognosis even without adjuvant chemotherapy $[13,14]$.

A recent study of TNBCs has revealed at least 6 distinct subtypes defined by their gene expression profiles [15]. These subtypes differ in important biological pathways and prognosis. The subtypes identified were termed basal-like 1 and 2, immunomodula-

\section{KARGER \\ Fax +497614520714

\section{(๑) 2015 S. Karger GmbH, Freiburg}

$1661-3791 / 15 / 0103-0159 \$ 39.50 / 0$
PD Dr. Frederik Marmé 
tory (IM), mesenchymal (M), mesenchymal stem-like (MSL) and luminal androgen receptor (LAR) based on gene ontology analysis. Within these subtypes, there seem to be some potentially targetable driver pathways, which could be exploited for future therapeutic strategies. Prognosis varies within these TNBC subtypes ranging from a median overall survival (OS) of 5.7 month for LAR to 24.8 months for the IM subtype [16]. However, these data, derived from an analysis of The Cancer Genome Atlas (TCGA) data, are still limited by relatively small numbers.

Evidence-based specific therapies for TNBC or even subtypespecific therapies are currently not yet available, with the exception maybe of platinum salts for patients carrying deleterious $B R C A$ mutations. This review summarizes the available evidence for specific targeted therapies for TNBC and attempts to give an outlook on future targeted therapeutic strategies.

\section{Platinum Salts}

It might appear strange to comment on a chemotherapeutic agent in a review addressing targeted therapies for TNBC. However, the mode of action of platinum salts at least in BRCA-deficient tumours is similar to a targeted agent. The strong interest in platinum-based therapies in TNBC is based on phenotypic similarities between BRCA1-associated breast cancer and triple-negative disease or more precisely the basal-like subtype. Roughly $80 \%$ of $B R C A 1$-associated tumours are basal like. However, the majority of basal-like tumours are not BRCA associated but sporadic. The shared phenotype has led to the speculation that sporadic basal-like tumours might also share defects in homologous recombination (HR) with their $B R C A$-associated counterparts, albeit caused by different mechanisms like, for example, $B R C A$ methylation and might, therefore, have a similar sensitivity to platinum salts. Platinumbased chemotherapy leads to intra- and inter-strand cross-links, which result in double-strand breaks during replication. These require HR as an error-free DNA-repair mechanism. If a cells harbours HR defects, error-prone compensatory repair mechanisms step in and lead to a high degree of genomic instability, finally leading to the death of the tumour cell. Preclinical data pointed to an extraordinary sensitivity of $B R C A$-associated breast and ovarian cancers to platinum agents. However, it took a long time until randomized trials provided first evidence that at least a subgroup of TNBC patients might specifically benefit from platinum-based chemotherapy. Several studies in unselected TNBCs revealed discouraging results. For example, the BALI-1 study, a randomized phase II trial that mainly randomized first- and second-line metastatic patients with TNBC to either cisplatin or the combination of cisplatin and cetuximab only yielded an objective response rate (ORR) of $10 \%$ for the monotherapy with a median progression-free survival (PFS) of 1.5 months [17]. A further randomized phase II trial, the TBCRC001 study, demonstrated an ORR of 17\% for the combination of carboplatin and cetuximab [18]. The TBCRC009, a single-arm phase II trial $(\mathrm{n}=86)$ demonstrated an ORR of $25.6 \%$ for carbo- or cisplatin in unselected first- and second-line TNBCs, which is comparable to responses that can be expected to other chemotherapeutic agents [19]. However, in an exploratory analysis, the ORR was considerably higher in $B R C A$-associated tumours than in sporadic TNBCs (54.5 vs. 19.7\%). This did not, however, result in a prolonged PFS or OS in this small study.

None of these data suggest that there is a superior efficacy of platinum agents in unselected TNBCs. The largest and thus far only trial directly comparing a platinum-based therapy to a taxane in metastatic breast cancer (MBC) has just been presented [20]. The TNT trial randomized 376 patients with metastatic TNBC to either carboplatin or docetaxel. Patients were encouraged to cross over to the alternative monotherapy upon progression if appropriate. There were no significant differences in terms of ORR, PFS and OS during the randomized phase or the cross-over phase in the overall study population. However, an exploratory analysis revealed a significant benefit from carboplatin over docetaxel in BRCA $1 / 2$ mutation carriers, with an ORR of $68 \%$ versus $33 \%$ and a PFS of 6.8 months versus 4.8 months. A test for interaction between $B R C A$ status and therapy was positive. Further preliminary data from the TNT trial suggest that docetaxel might be superior to carboplatin in the non-basal-like subgroup defined by PAM50 [20]. Although these data do not suggest a greater efficacy of platinum agents in unselected TNBCs, they do point to a role in BRCAassociated breast cancer. A small prospective single-arm phase II trial including $20 \mathrm{BRCA} 1$ mutation carriers with $\mathrm{MBC}$ who were treated with single-agent cisplatin $\left(75 \mathrm{mg} / \mathrm{m}^{2} \mathrm{q} 3 \mathrm{w}\right)$ demonstrated an ORR of $80 \%$ with a PFS of 12 months [21].

Two prospective randomized neoadjuvant trials in TNBC, the GeparSixto trial and the CALGB 40603 trial reported an increase in pCR rate (ypT0/is ypN0) of $10.5 \%$ and $13 \%$ through the addition of carboplatin $[22,23]$. Preliminary data from the GeparSixto trial suggest that this gain in pCR rate is mainly driven by $B R C A$-associated TNBCs, for which the addition of carboplatin increased the pCR rate by $23 \%$ [24]. It is still unclear if and how this increase in pCR rate will impact survival, but the data add to the evidence of a specific benefit from platinum agents in BRCA-associated TNBCs. The current AGO guidelines breast cancer version 2015.1D support the use of platinum agents at least in BRCA-associated TNCB (and patients with a positive family history of breast or ovarian cancer) with a single $(+)$ (www.ago-online.de).

\section{PARP Inhibitors}

HR represents an important error-free DNA repair mechanism for double-strand breaks. In a process involving BRCA1/2 the homologous sequence of the sister chromatid is used to precisely repair the double-strand break. In $B R C A$-associated tumours the non-mutated $B R C A 1 / 2$ allele is inactivated, leading to the accumulation of double-strand breaks and genomic instability. Inhibition of base excision repair (BER) in such cells leads to the accumulation of double-strand breaks during replication, which would usually rely on BRCA-mediated HR. Poly(ADP-ribose) polymerase (PARP) is an enzyme centrally involved in BER by recruiting the 
Table 1. Current phase III trials of PARP inhibitors in breast cancer

\begin{tabular}{|c|c|c|c|c|c|}
\hline Sponsor & $\begin{array}{l}\text { ClinicalTrial.gov } \\
\text { identifier }\end{array}$ & Trial & Treatment & Population & Biomarker \\
\hline Abbvie & NCT02032277 & Brightness & $\begin{array}{l}\text { carboplatin based NAC + } \\
\text { veliparib/placebo }\end{array}$ & $\begin{array}{l}\text { triple-negative early } \\
\text { breast cancer }\end{array}$ & - \\
\hline AstraZeneca & NCT02032823 & OlympiA & $\begin{array}{l}\text { maintainance } \\
\text { olaparib/placebo }\end{array}$ & $\begin{array}{l}\text { HER2 }{ }^{-} \text {early breast } \\
\text { cancer }\end{array}$ & $\begin{array}{l}\text { BRCA1/2 } \\
\text { mutation }\end{array}$ \\
\hline AstraZeneca & NCT02000622 & OlympiaD & $\begin{array}{l}\text { olaparib vs. physician's } \\
\text { choice }\end{array}$ & $\begin{array}{l}\text { advanced/metastatic } \\
\text { HER2- breast cancer }\end{array}$ & $\begin{array}{l}\text { BRCA } 1 / 2 \\
\text { mutation }\end{array}$ \\
\hline Abbvie & NCT02163694 & Brocade & $\begin{array}{l}\text { carboplatin/paclitaxel } \\
\text { plus veliparib/placebo }\end{array}$ & $\begin{array}{l}\text { advanced/metastatic } \\
\text { HER2- breast cancer }\end{array}$ & $\begin{array}{l}\text { BRCA1/2 } \\
\text { mutation }\end{array}$ \\
\hline Tesaro & NCT01905592 & BRAVO & $\begin{array}{l}\text { niraparib vs. physician's } \\
\text { choice }\end{array}$ & $\begin{array}{l}\text { advanced/metastatic } \\
\text { HER2 }{ }^{-} \text {breast cancer }\end{array}$ & $\begin{array}{l}\text { BRCA1/2 } \\
\text { mutation }\end{array}$ \\
\hline BioMarin & NCT01945775 & EMBRACA & $\begin{array}{l}\text { talazoparib vs. physician's } \\
\text { choice }\end{array}$ & $\begin{array}{l}\text { advanced/metastatic } \\
\text { HER2- breast cancer }\end{array}$ & $\begin{array}{l}\text { BRCA } 1 / 2 \\
\text { mutation }\end{array}$ \\
\hline
\end{tabular}

BER machinery to single-strand breaks. Inhibiting PARP in HRdeficient cells leads to the accumulation of double-strand breaks, genomic instability and specific synthetic lethality [25].

Again, phenotypic similarities between TNBCs as a surrogate for the basal-like subtype and $B R C A$-associated breast cancers have led to the strategy of selecting patients for PARP inhibition on the basis of their TNBC phenotype [26]. Other investigators, however, have chosen to restrict the development of PARP inhibitors to $B R C A$-associated breast and other solid cancer types, which leaves a considerably smaller target population. Currently, several PARP inhibitors such as olaparib, veliparib, rucaparib, niraparib, talazoparib (BMN673) and others are undergoing clinical development. Olaparib was the first PARP inhibitor to be granted regulatory approval for recurrent high-grade serous ovarian cancer by the US Food and Drug Administration (FDA) and the European Medicines Agency (EMEA). Olaparib was first developed in a singlearm phase II study recruiting patients with $B R C A$-associated breast cancer in 2 consecutive cohorts treated with $100 \mathrm{mg}$ twice daily (bid) and $400 \mathrm{mg}$ bid single-agent olaparib, respectively. Results of this trial demonstrated a promising dose-dependent ORR of $22 \%$ (100 mg bid) and 41\% (400 mg bid) with a median PFS of 5.7 months for the higher dose [27]. A parallel trial in BRCA-associated ovarian cancer showed comparable results [28].

Gelmon et al. [29] studied the efficacy of olaparib in unselected TNBCs not restricted to BRCA-associated breast cancer and unselected serous ovarian cancer. Their study included 26 patients with TNBC but was unable to demonstrate any responses as defined by RECIST (Response Evaluation Criteria in Solid Tumors). In contrast to an ORR of $41 \%$ (BRCA mutated) and 24\% (BRCA wild type) seen in the ovarian cancer cohort, no confirmed responses were seen among the 26 breast cancer patients. A recent study including several BRCA-associated solid tumours demonstrated a discouraging ORR of only $12.9 \%$ with a PFS of only 3.7 months in the 62 BRCA-associated breast cancer patients included. These patients were heavily pre-treated with a mean of 4.6 prior regimens for advanced disease. Two thirds had also received prior platinumbased therapy. The ORR seemed to be higher in breast cancer pa- tients without prior platinum chemotherapy (20\% vs. 9.5\%). However, the 193 BRCA-associated ovarian cancers included in this study had all received prior platinum chemotherapy and were considered to be platinum resistant or not suitable for platinum therapy, yet still had an ORR of $31 \%$ and a PFS of 7.0 months. Overall, these data suggest that PARP inhibition by olaparib is more effective in (BRCA-associated) ovarian cancer than in BRCA-associated breast cancer. A cross-trial comparison might suggest a superior efficacy of olaparib in the trial by Tutt et al. [27], which demonstrated an ORR of $41 \%$ in the $400 \mathrm{mg}$ bid cohort. Whether this is due to a lesser extent of pre-treatment and fewer patients with prior platinum chemotherapy is a matter of speculation. Trials attempting to combine olaparib with paclitaxel failed to achieve a meaningful dose intensity of paclitaxel because of high rates of neutropenia despite G-CSF (granulocyte colony-stimulating factor) prophylaxis.

As part of the I-Spy 2 trial the combination of carboplatin and veliparib added to weekly paclitaxel and followed by doxorubicin/ cyclophosphamide led to a doubling of the pCR rate in the triplenegative study population (from $26 \%$ to $52 \%$ ). Trial statistics in a phase III trial predicted a probability of success of $90 \%$ for this combination [30]. Table 1 summarizes current phase III trials investigating PARP inhibitors in breast cancer.

Several mechanisms of PARP inhibitor resistance in $B R C A$-associated tumours have been proposed. These include genetic reversal of truncating mutations, stabilisation of mutated BRCA proteins, e.g. by HSP90 (possibly targetable by HSP90 inhibitors), loss of 53BP1, hypomorphic BRCA function and drug efflux pumps [31]. Several strategies to overcome these resistance mechanisms are currently under investigation. The exploration of these mechanisms as potential predictive factors is also warranted.

\section{Anti-Angiogenesis}

TNBC is associated with a significantly higher expression and more frequent amplification of vascular endothelial growth factor 
(VEGF)-A [32, 33]. This has led to the hypothesis of a specifically higher activity of anti-angiogenic drugs in TNBC. Several antiangiogenic agents are currently under development. Bevacizumab, a monoclonal anti-VEGF-A antibody is the only drug currently approved for the treatment of MBC by the EMEA, but not by the FDA.

In the pivotal open-label randomized phase III trial, E2100, in first-line unselected MBC, the addition of bevacizumab to weekly paclitaxel increased median PFS from 5.9 to 11.8 months (hazard ratio $0.6, \mathrm{p}<0.001)$ and doubled the response rate $(49.2 \%$ vs. $25.2 \%, \mathrm{p}<0.001)$. However there was no significant improvement in OS $[34,35]$.

Two further first-line phase III studies (AVADO and RIBBON-1) confirmed the benefit of the addition of bevacizumab to first-line chemotherapy, although to a lesser extent [36-38]. The efficacy of bevacizumab in addition to weekly paclitaxel could further be confirmed by similar results observed for the combination in the TURANDOT study (PFS 11 months, ORR 44\%) and CALGB 40502 study (PFS 10.6 months) [39, 40].

However, neither of these individual randomized trials nor a joint analysis was able to demonstrate a significant OS benefit in unselected patients. A trend towards an improved OS for the TNBC cohort (17.9 vs. 12.6 months, $\mathrm{p}=0.0534)$, in addition to a significant PFS benefit (6.0 vs. 2.7 months; hazard ratio 0.45 ; $\mathrm{p}=0.0006$ ), was observed only in an explorative subgroup analysis of the randomized phase III second-line trial, RIBBON-2 [41]. However, this can at best be regarded as a hypothesis-generating retrospective analysis due to small number of patients $(\mathrm{n}=159)$ and statistical considerations. Bevacizumab is not approved for second-line treatment.

The important question addressing the hypothesis of a specific bevacizumab benefit in TNBCs was addressed in a joint subgroup analysis of all TNBCs within the 3 randomized phase III first-line trials. This analysis included 621 patients with TNBC from these trials and confirmed the increased ORR ( $42 \%$ vs. $23 \%$ ) and PFS (8.1 vs. 5.4 months; hazard ratio 0.63 ; $\mathrm{p}<0.0001$ ), although without a trend for an improved OS (18.9 vs. 17.5 months; hazard ratio 0.96; ns) [42].

The joint analysis only found a significantly improved OS for triple-negative patients who had previously received a taxane-containing adjuvant regimen (25.6 vs. 15.0 months; hazard ratio 0.61 , 95\% confidence interval 0.4-0.94). However, this was only an explorative subgroup analysis of a subgroup analysis.

Data from a large adjuvant phase III trial exclusively run in TNBC, the BEATRICE trial $(\mathrm{n}=2,591)$, did not demonstrate an improvement in DFS or OS for the addition of bevacizumab to adjuvant chemotherapy followed by bevacizumab maintenance therapy [43].

Data from neoadjuvant studies are inconsistent with respect to a specific benefit from bevacizumab in TNBCs, some demonstrating a small increase in pCR rates in ER-positive cases, others in the TNBC population $[22,44,45]$.

In summary, the effect of bevacizumab in TNBCs is similar to that seen in unselected patients. However, because of limited thera- peutic options and frequent aggressive disease dynamics, bevacizumab might play a special role in the treatment of metastatic TNBC. Higher response rates and longer PFS can more effectively counteract and control existing or threatening symptoms without the need for polychemotherapy with all its toxicities.

One of the main drawbacks for anti-angiogenic therapies including bevacizumab is the lack of a clinically validated predictive biomarker. Triple-negative status does not play a role in predicting benefit in this respect. Currently, a prospective randomized trial, the Meridian trial (NCT01663727), is investigating the role of serum VEGF-A as a potential factor for patient selection for bevacizumab.

In addition to monoclonal antibodies, a series of tyrosine kinase inhibitors (TKIs) against pro-angiogenic kinases like receptors for VEGF and PDGF have been developed, e.g. sunitinib, sorafenib and pazopanib. Because of the increased off-target effects of these TKIs, combination with chemotherapeutic agents has proven difficult. Their efficacy as monotherapy in MBC is limited with ORRs ranging from $0 \%$ to $11 \%$ [46-50].

Sunitinib and sorafenib have been developed in phase IIb/III programmes. Sunitinib was unable to provide any benefit either as monotherapy compared to capecitabine or in combination with chemotherapy $[49,51-53]$. There are no specific data for triplenegative patients from these trials. Sorafenib demonstrated activity in a phase IIb study in combination with either capecitabine or gemcitabine, although at the cost of a high rate of palmar-plantar erythema (45\% grade 3) [54]. In the light of their modest activity but considerable toxicity, it is unlikely that anti-angiogenic TKIs will play an important role in the treatment of $\mathrm{MBC}$, regardless of subtype.

\section{Epidermal Growth Factor Receptor}

Overexpression of epidermal growth factor receptor (EGFR) can be observed in more than $50 \%$ of TNBCs and is correlated with a poor prognosis $[55,56]$. This observation prompted a series of clinical trials incorporating anti-EGFR directed therapies, such as cetuximab and lapatinib.

The TBCRC001 trial investigated the activity of cetuximab, a monoclonal anti-EGFR antibody as a single agent and in combination with carboplatin. Single-agent cetuximab demonstrated a discouraging ORR of $6 \%$ with a clinical benefit rate of only $10 \%$. The combination with carboplatin resulted in an ORR of $17 \%$. The median time to progression for single-agent cetuximab was only 1.7 months and 2.6 months for the combination. However, despite these disappointing results, 4 patients ( 2 in the monotherapy and 2 in the combination arm) in the trials had long-lasting responses of more than 12 months (2 even over 2.5 years) [18]. Results from serial biopsies available for 18 patients within the trial showed that $81 \%$ of the patients demonstrated EGFR-pathway activation; however, this could only be inhibited by cetuximab in 5 of the patients (38\%). Clinical benefit was restricted to these patients [18]. Thus, apart from EGFR overexpression, downstream activation of the 
Table 2. Results from selected trials with EGFR-targeted therapies including TNBCs

\begin{tabular}{|c|c|c|c|c|c|c|c|c|c|c|c|c|}
\hline Study [Ref] & Population & Regimen & $\mathrm{n}$ & ORR & OR & $p-$ & $\begin{array}{l}\text { PFS/ } \\
\text { TTP* }^{*}\end{array}$ & $\mathrm{hr}$ & $p-$ & OS & $\mathrm{hr}$ & $p-$ \\
\hline \multirow{2}{*}{$\begin{array}{r}\text { BALI-1 } \\
{[17]}\end{array}$} & \multirow[t]{2}{*}{ mTNBC } & cisplatin & 58 & 10.3 & & & 1.5 & & & 9.4 & & \\
\hline & & cisplatin + cetuximab & 115 & 20.0 & 2.13 & 0.11 & 3.7 & 0.67 & 0.032 & 12.9 & 0.82 & 0.31 \\
\hline \multirow{3}{*}{$\begin{array}{c}\text { TBCRC001 } \\
{[18]}\end{array}$} & \multirow[t]{3}{*}{ mTNBC } & cetuximab & 31 & 6 & & & $1.4^{*}$ & & & 7.5 & & \\
\hline & & PD $\rightarrow$ carboplatin + cetuximab & $25 / 31$ & 16 & & & $2.6^{*}$ & & & - & & \\
\hline & & carboplatin + cetuximab & 71 & 17 & & & $2.1^{*}$ & & & 10.4 & & \\
\hline \multirow{4}{*}{$\begin{array}{l}\text { USON } 225200 \\
\quad[60]\end{array}$} & \multirow[t]{2}{*}{ MBC } & irinotecan/carboplatin & 75 & 31 & & & 4.6 & & & 12.3 & & \\
\hline & & irinotencan/carboplatin/cetuximab & 79 & 38 & & & 4.8 & & & 12.6 & & \\
\hline & \multirow[t]{2}{*}{ mTNBC } & irinotecan/carboplatin & 33 & 30 & & & 5.1 & & & 12.3 & & \\
\hline & & irinotencan/carboplatin/cetuximab & 39 & 49 & & & 4.7 & & & 15.5 & & \\
\hline \multirow{6}{*}{$\begin{array}{r}\text { EGF30001 } \\
{[57,58]}\end{array}$} & \multirow[t]{2}{*}{ HER2+ MBC } & paclitaxel & 37 & 37.8 & & & 5.0 & & & 19.2 & & \\
\hline & & paclitaxel/lapatinib & 49 & 63.3 & 3.0 & 0.023 & 8.1 & 0.52 & 0.004 & 24.4 & & \\
\hline & \multirow[t]{2}{*}{ HER2- MBC } & paclitaxel & 204 & 23.5 & & & 5.4 & & & 20.3 & & \\
\hline & & paclitaxel/lapatinib & 202 & 30.2 & 1.4 & 0.13 & 5.4 & 1.10 & 0.39 & 23.1 & 0.89 & 0.44 \\
\hline & \multirow[t]{2}{*}{ mTNBC } & paclitaxel & 60 & - & & & 4.6 & & & - & & \\
\hline & & paclitaxel/lapatinib & 71 & - & & & 4.8 & 1.25 & 0.26 & - & & \\
\hline
\end{tabular}

EGFR = epidermal growth factor receptor, TNBC = triple-negative breast cancer, $\mathrm{mTNBC}=$ metastatic TNBC, $\mathrm{ORR}=$ objective response rate, $\mathrm{PFS}=$ progression - free survival, $\mathrm{hr}=$ hazard ratio, $\mathrm{TTP}=$ time to progression, $\mathrm{MBC}=$ metastatic breast cancer.

pathway might play an important role. The largest published randomized study so far, the BALI-1 trial including 173 patients with metastatic TNBC, $73 \%$ in a first-line setting, randomized patients to either a combination of cetuximab and cisplatin or cisplatin alone. There was a significant benefit in terms of PFS for the combination ( 3.7 vs. 1.5 months; hazard ratio $0.675 ; \mathrm{p}=0.032$ ) and a non-significant improvement in response rate $(20 \%$ vs. $10.3 \%$; $\mathrm{p}=0.11)$ and OS (12.9 vs. 9.4 months; hazard ratio $0.82 ; \mathrm{p}=0.31$ ). A possible impact of the $53 \%$ of patients crossing over to cetuximab containing regimens at progression remains a matter of speculation [17].

In addition, there are no data on activation of EGFR signalling (e.g. EGFR expression, etc.) or KRAS status and downstream activation of the pathway, which like in colorectal cancer could impact the efficacy of cetuximab in MBC. Currently, panitumumab, a human monoclonal anti-EGFR antibody is under investigation in combination with carboplatin and gemcitabin in TNBC (NCT00894504). EGFR-targeted TKIs have also been investigated in MBC. Lapatinib, a dual EFGR and HER2 inhibitor was initially studied in a randomized phase III trial in combination with firstline paclitaxel compared to paclitaxel alone. Analysis of the 131 TNBC patients included in this trial did not show a benefit from the addition of lapatinib to paclitaxel in first-line metastatic TNBC (median PFS 4.6 vs. 4.8 months, $\mathrm{p}=0.255$ ) and activity was not dependent on EGFR expression [57, 58]. Further, EGFR-directed TKIs have been investigated in clinical trials for benefit in TNBC, e.g. erlotinib in combination with carboplatin and docetaxel (NCT00491816) and gefitinib.

Concluding, preclinical and translational research suggests that a considerable proportion of TNBCs are dependent on EGFR signalling. Clinical data point to a modest effect of EGFR targeted therapies at least in a subset of TNBCs. This subset of TNBC should be more precisely defined by clinically validated predictive factors. In many cases constitutive EGFR-pathway activation seems to be at least partially due to downstream activation of the pathway, e.g. through KRAS amplification/mutation or CRYAB expression $[18,59]$. Successful targeted therapies in these cases will require different strategies. Table 2 gives a summary of results from selected trials.

\section{Further Experimental Therapeutic Targets}

\section{Immune Checkpoint Inhibitors}

Rapid progression and the development of resistance pose the main challenges in the treatment of TNBC. Immunotherapeutic approaches may offer one strategy to circumvent these problems. TNBCs harbour more non-synonymous mutations compared to luminal-type breast cancers and more tumour-infiltrating lymphocytes, which in TNBC have a strong predictive and prognostic meaning. The non-synonymous mutations may act as neoantigens that could principally be recognized by the host immune system. The ability of neoantigens to elicit an immune response is dependent on their ability to form stable complexes with major histocompatibility complex (MHC) I molecules and therefore to be presented and recognized by specific T-cell receptors. Whereas the mere presence of non-synonymous mutations is not prognostic in several types of cancers, immunogenic neoantigens as predicted by validated algorithms are independent prognostic factors [61]. Tumours exploit several mechanisms to evade the recognition or destruction by the host immune system. Amongst them is the downregulation of MHC I molecules. Further, CTLA-4 and PD-1 (programmed death receptor 1 ) are 2 key cell-surface receptors that dampen $\mathrm{T}$-cell response by triggering inhibitory pathways 
when bound by their ligands. For the PD-1 pathway, ligand expression (PD-L1) frequently occurs on tumours cells, which directly leads to death of reactive $\mathrm{T}$ cells. Current therapeutic strategies to block these immune checkpoints mostly engage CTLA-4, PD-1 and PD-L1. Ipilimumab, a monoclonal anti-CTLA-4 antibody, nivolumab and pembrolizumab (monoclonal antibodies against PD-1) have been approved for the treatment of several solid tumours and have led to prolonged responses. First data to suggest that such immune checkpoint inhibition strategies might be successful in TNBC were presented at the San Antonio Breast Cancer Symposium in December 2014. Pembrolizumab demonstrated an ORR of $18.5 \%$ in a phase Ib study $(\mathrm{n}=32)$ in PD-L1positive TNBCs, most of which were heavily pretreated. Response rates were independent of the number of prior lines of therapy. Amongst those patients responding, responses were long lasting, with the median duration of response not reached at the time of presentation [62]. Inhibition of PD-L1 by the monoclonal antiPD-L1 antibody MPDL3280A in PD-L1-positive TNBCs $(\mathrm{n}=12)$ yielded an ORR of $33 \%$ in a phase Ia trial, again with long-lasting responses [63]. The duration of response seen in these heavily pretreated patients with metastatic TNBC is extraordinary and encouraging. Immune checkpoint blockade might in fact constitute the most promising of targeted therapies for TNBC currently being investigated.

\section{Androgen Receptor}

A gene expression study in 99 breast cancer cases, including 41 TNBC, demonstrated that a subgroup of TNBCs was grouped together with ER-positive cases in a cluster analysis. Further investigations revealed that, despite the lack of ER and PgR expression, these cases show an expression of an ER-regulated gene set. This is caused by the expression of the androgen receptor (AR) in these cases. TNBC cell lines with the respective phenotype respond to treatment with androgens and their antagonists. This constitutes a rationale for anti-androgenic therapies in these patients, which likely correspond to the LAR subtype proposed by Lehmann and colleagues [15]. The androgen antagonist bicalutamide is currently being investigated in a phase II trial, the TBCRC011 trial (NCT00468715). Further studies of the selective, irreversible Cyp17 inhibitor abiraterone in ER-positive and ER-negative ARexpressing MBC are under way (NCT00755885).

\section{Src Inhibitors}

Src is centrally involved in signal transduction of several growth factor receptors and promotes proliferation and invasiveness [64]. Gene expression studies and preclinical data suggested that basallike breast cancers might be particularly sensitive against inhibition of Src by the dual Abl/Src inhibitor dasatinib $[65,66]$. However, a phase II study of dasatinib in TNBC reported a clinical benefit rate of only $9.2 \%$ and studies in ER-positive disease only demonstrated a very modest activity with a disease control rate of $19 \%[65,67]$.
Dasatinib as a single agent without further patient selection is ineffective in TNBC. A current trial is investigating a gene expression profile as a predictive marker for response to dasatinib.

\section{PI3K/Akt/mTOR}

Loss of PTEN expression leading to an activation of Akt/mTOR signalling is a quite frequent observation in TNBC $[32,68]$. Phosphorylation of mTOR in early TNBC is associated with an adverse prognosis $[69,70]$. Several trials including mTOR inhibitors in TNBC are currently under way. A randomized neoadjuvant study adding everolimus to weekly paclitaxel followed by fluorouracilepirubicin-cyclophosphamide (FEC) in 62 patients with TNBC led to a non-significant increase in pCR rate from $25.9 \%$ to $30.4 \%$ $(\mathrm{p}=0.76)$ with the addition of everolimus. However, the results are strongly limited by the small numbers. The large neoadjuvant GeparQuinto trial, randomized HER2-negative patients not responding to 4 cycles of EC to everolimus plus paclitaxel or paclitaxel alone. Everolimus did not lead to an improved pCR (3.6\% vs. 5.6\%). However, as the trial included ER-positive patients and selected only the resistant population to everolimus versus control, no conclusions can be drawn with respect to early TNBC in general [71].

Balko et al. [72] used a different approach to identify possible important therapeutic targets in TNBC. Considering that the poor prognosis of TNBC is mainly due to those patients not responding to (neo)adjuvant chemotherapy (NACT), they analysed the residual tumours after NACT, speculating that these would reflect biology of the resistant proportion of the tumour finally responsible for distant metastases and death. Performing next generation sequencing, gene expression analysis and copy number variation analysis on 111 residual TNBCs after NACT, they were able to identify potentially targetable alterations in $90 \%$ of patients. MYC amplification and MEK activation frequently co-occurred and had a significant interaction with respect to prognosis. Pre-clinical models demonstrated an interesting activity of MEK inhibitors in respective tumours. Interestingly, JAK2 amplification found in residual tumours was associated with in poor prognosis in a multivariate analysis (hazard ratio $4.16, \mathrm{p}=0.002$ ). Even though these results have to be validated, ruxolitinib, a JAK2 inhibitor, is currently approved for the treatment of myelofibrosis.

In conclusion, current therapy for TNBC is mainly analogous to unselected $\mathrm{MBC}$, maybe with the exception of platinum in BRCAassociated tumours. There is still no targeted therapy with specific activity in TNBC. However, the role of PARP inhibitors and immunotherapies might lead to future improvements, as might targeted therapies directed against the resistant residual tumour after NACT.

\section{Disclosure Statement}

F. Marmé received honoraria from Roche, Novartis, Celgene, Eisai, AstraZenca and Amgen. 


\section{References}

1 von Minckwitz G, Untch M, Nüesch E, et al.: Impact of treatment characteristics on response of different breast cancer phenotypes: Pooled analysis of the German neo-adjuvant chemotherapy trials. Breast Cancer Res Treat 2011;125:145-156.

2 Bauer KR, Brown M, Cress RD, et al.: Descriptive analysis of estrogen receptor (ER)-negative, progesterone receptor (PR)-negative, and HER2-negative invasive breast cancer, the so-called triple-negative phenotype: A population-based study from the California Cancer Registry. Cancer 2007;109:1721-1728.

3 Dent R, Trudeau M, Pritchard KI, et al.: Triple-negative breast cancer: Clinical features and patterns of recurrence. Clin Cancer Res 2007;13:4429-4434.

4 Gluz O, Liedtke C, Gottschalk N, et al.: Triple-negative breast cancer-current status and future directions. Ann Oncol 2009;20:1913-1927.

5 Bosch A, Eroles P, Zaragoza R, et al.: Triple-negative breast cancer: Molecular features, pathogenesis, treatment and current lines of research. Cancer Treat Rev 2010;36:206-215.

6 de Ruijter TC, Veeck J, de Hoon JP, et al.: Characteristics of triple-negative breast cancer. J Cancer Res Clin Oncol 2011;137:183-192.

7 Liedtke C, Mazouni C, Hess KR, et al.: Response to neoadjuvant therapy and long-term survival in patients with triple-negative breast cancer. J Clin Oncol 2008;26:1275-1281.

8 Jatoi I, Anderson WF, Jeong JH, Redmond CK: Breast cancer adjuvant therapy: Time to consider its time-dependent effects. J Clin Oncol 2011;29:2301-2304.

9 von Minckwitz G, Untch M, Blohmer JU, et al.: Definition and impact of pathologic complete response on prognosis after neoadjuvant chemotherapy in various intrinsic breast cancer subtypes. J Clin Oncol 2012;30: 1796-1804.

10 Cortazar P, Zhang L, Untch M, et al.: Pathological complete response and long-term clinical benefit in breast cancer: The CTNeoBC pooled analysis. Lancet 2014;384:164-172.

11 Heitz F, Harter P, Lueck HJ, et al.: Triple-negative and HER2-overexpressing breast cancers exhibit an elevated risk and an earlier occurrence of cerebral metastases. Eur J Cancer 2009;45:2792-2798.

12 Lin NU, Claus E, Sohl J, et al.: Sites of distant recurrence and clinical outcomes in patients with metastatic triplenegative breast cancer: High incidence of central nervous system metastases. Cancer 2008;113:2638-2645.

13 Cadoo KA, McArdle O, O’Shea AM, et al.: Management of unusual histological types of breast cancer. Oncologist 2012;17:1135-1145.

14 Huober J, Gelber S, Goldhirsch A, et al.: Prognosis of medullary breast cancer: Analysis of 13 international breast cancer study group (IBCSG) trials. Ann Oncol 2012;23:2843-2851.

15 Lehmann BD, Bauer JA, Chen X, et al.: Identification of human triple-negative breast cancer subtypes and preclinical models for selection of targeted therapies. J Clin Invest 2011;121:2750-2767.

16 Mayer IA, Abramson VG, Lehmann BD, Pietenpol JA New strategies for triple-negative breast cancer-deciphering the heterogeneity. Clin Cancer Res 2014;20: 782-790.

17 Baselga J, Gomez P, Greil R, et al.: Randomized phase II study of the anti-epidermal growth factor receptor monoclonal antibody cetuximab with cisplatin versus cisplatin alone in patients with metastatic triple-negative breast cancer. J Clin Oncol 2013;31:2586-2592.

18 Carey LA, Rugo HS, Marcom PK, et al.: TBCRC 001: Randomized phase II study of cetuximab in combination with carboplatin in stage IV triple-negative breast cancer. J Clin Oncol 2012;30:2615-2623.
19 Isakoff SJ, Mayer EL, He L, et al.: TBCRC009: A multicenter phase II clinical trial of platinum monotherapy with biomarker assessment in metastatic triple-negative breast cancer. J Clin Oncol 2015; [Epub ahead of print].

20 Tutt A, Ellis P, Kilburn L, et al.: Abstract s3-01: The TNT trial: A randomized phase III trial of carboplatin (C) compared with docetaxel (D) for patients with metastatic or recurrent locally advanced triple negative or BRCA1/2 breast cancer (CRUK/07/012). Cancer Res 2015;75:S3-01.

21 Byrski T, Dent R, Blecharz P, et al.: Results of a phase II open-label, non randomized trial of cisplatin chemotherapy in patients with BRCA1-positive metastatic breast cancer. Breast Cancer Res 2012;14:R110.

22 Sikov WM, Berry DA, Perou CM, et al.: Impact of the addition of carboplatin and/or bevacizumab to neoadjuvant once-per-week paclitaxel followed by dosedense doxorubicin and cyclophosphamide on pathologic complete response rates in stage II to III triplenegative breast cancer: CALGB 40603 (alliance). J Clin Oncol 2015;33:13-21.

23 von Minckwitz G, Schneeweiss A, Loibl S, et al.: Neoadjuvant carboplatin in patients with triple-negative and HER2-positive early breast cancer (GeparSixto; GBG 66): A randomised phase 2 trial. Lancet Oncol 2014;15:747-756.

24 Von Minckwitz G, Hahnen E, Fasching PA, et al.: Pathological complete response (pCR) rates after carboplatin-containing neoadjuvant chemotherapy in patients with germline BRCA (gBRCA) mutation and triple-negative breast cancer (TNBC): Results from GeparSixto. ASCO Meeting Abstracts 2014;32:1005

25 Farmer H, McCabe N, Lord CJ, et al.: Targeting the DNA repair defect in BRCA mutant cells as a therapeutic strategy. Nature 2005;434:917-921

26 Turner N, Tutt A, Ashworth A: Hallmarks of 'BRCAness' in sporadic cancers. Nat Rev Cancer 2004;4:814819.

27 Tutt A, Robson M, Garber JE, et al.: Oral poly(ADPribose) polymerase inhibitor olaparib in patients with BRCA 1 or BRCA 2 mutations and advanced breast cancer: A proof-of-concept trial. Lancet 2010;376: 235-244.

28 Audeh MW, Carmichael J, Penson RT, et al.: Oral poly(ADP-ribose) polymerase inhibitor olaparib in patients with BRCA1 or BRCA2 mutations and recurrent ovarian cancer: A proof-of-concept trial. Lancet 2010; 376:245-251.

29 Gelmon KA, Tischkowitz M, Mackay H, et al.: Olaparib in patients with recurrent high-grade serous or poorly differentiated ovarian carcinoma or triple-negative breast cancer: A phase 2, multicentre, open-label, nonrandomised study. Lancet Oncol 2011;12:852-861.

30 Rugo H, Olopade O, DeMichele A, et al.: Abstract s502: Veliparib/carboplatin plus standard neoadjuvant therapy for high-risk breast cancer: First efficacy results from the I-SPY 2 trial. Cancer Res 2013;73:S5-02.

31 Bouwman P, Jonkers J: Molecular pathways: How can BRCA-mutated tumors become resistant to PARP inhibitors? Clin Cancer Res 2014;20:540-547.

32 Andre F, Job B, Dessen P, et al.: Molecular characterization of breast cancer with high-resolution oligonucleotide comparative genomic hybridization array. Clin Cancer Res 2009;15:441-451.

33 Linderholm BK, Hellborg H, Johansson U, et al.: Significantly higher levels of vascular endothelial growth factor (VEGF) and shorter survival times for patients with primary operable triple-negative breast cancer. Ann Oncol 2009;20:1639-1646.

34 Miller K, Wang M, Gralow J, et al.: Paclitaxel plus bevacizumab versus paclitaxel alone for metastatic breast cancer. N Engl J Med 2007;357:2666-2676.
35 Gray R, Bhattacharya S, Bowden C, et al.: Independent review of E2100: A phase III trial of bevacizumab plus paclitaxel versus paclitaxel in women with metastatic breast cancer. J Clin Oncol 2009;27:4966-4972.

36 Miles DW, Chan A, Dirix LY, et al.: Phase III study of bevacizumab plus docetaxel compared with placebo plus docetaxel for the first-line treatment of human epidermal growth factor receptor 2-negative metastatic breast cancer. J Clin Oncol 2010;28:3239-3247.

37 Pivot X, Schneeweiss A, Verma S, et al.: Efficacy and safety of bevacizumab in combination with docetaxel for the first-line treatment of elderly patients with locally recurrent or metastatic breast cancer: Results from AVADO. Eur J Cancer 2011;47:2387-2395.

38 Robert NJ, Dieras V, Glaspy J, et al.: Ribbon-1: Randomized, double-blind, placebo-controlled, phase III trial of chemotherapy with or without bevacizumab for first-line treatment of human epidermal growth factor receptor 2-negative, locally recurrent or metastatic breast cancer. J Clin Oncol 2011;29:1252-1260.

39 Rugo HS, Barry WT, Moreno-Aspitia A, et al.: CALGB 40502/NCCTG N063H: Randomized phase III trial of weekly paclitaxel $(\mathrm{P})$ compared to weekly nanoparticle albumin bound nab-paclitaxel (NP) or ixabepilone (Ix) with or without bevacizumab (B) as first-line therapy for locally recurrent or metastatic breast cancer (MBC). J Clin Oncol 2012;30 Suppl; abstr CRA1002.

40 Lang I, Brodowicz T, Ryvo L, et al.: Bevacizumab plus paclitaxel versus bevacizumab plus capecitabine as first-line treatment for HER2-negative metastatic breast cancer: Interim efficacy results of the randomised, open-label, non-inferiority, phase 3 TURANDOT trial. Lancet Oncol 2013;14:125-133.

41 Brufsky A, Valero V, Tiangco B, et al.: Second-line bevacizumab-containing therapy in patients with triple-negative breast cancer: Subgroup analysis of the Ribbon-2 trial. Breast Cancer Res Treat 2012;133: 1067-1075.

42 Miles DW, Dieras V, Cortes J, et al.: First-line bevacizumab in combination with chemotherapy for HER2negative metastatic breast cancer: Pooled and subgroup analyses of data from 2447 patients. Ann Oncol 2013;24:2773-2780.

43 Cameron D, Brown J, Dent R, et al.: Adjuvant bevacizumab-containing therapy in triple-negative breast cancer (BEATRICE): Primary results of a randomised, phase 3 trial. Lancet Oncol 2013;14:933-942.

44 von Minckwitz G, Eidtmann H, Rezai M, et al.: Neoadjuvant chemotherapy and bevacizumab for HER2-negative breast cancer. N Engl J Med 2012;366:299-309.

45 Bear HD, Tang G, Rastogi P, et al.: Bevacizumab added to neoadjuvant chemotherapy for breast cancer. $\mathrm{N}$ Engl J Med 2012;366:310-320.

46 Cobleigh MA, Langmuir VK, Sledge GW, et al.: A phase I/II dose-escalation trial of bevacizumab in previously treated metastatic breast cancer. Semin Oncol 2003;30:117-124.

47 Bianchi G, Loibl S, Zamagni C, et al.: Phase II multicenter, uncontrolled trial of sorafenib in patients with metastatic breast cancer. Anticancer Drugs 2009;20: 616-624.

48 Moreno-Aspitia A, Morton RF, Hillman DW, et al.: Phase II trial of sorafenib in patients with metastatic breast cancer previously exposed to anthracyclines or taxanes: North central cancer treatment group and mayo clinic trial N0336. J Clin Oncol 2009;27:11-15.

49 Barrios CH, Liu MC, Lee SC, et al.: Phase III randomized trial of sunitinib versus capecitabine in patients with previously treated HER2-negative advanced breast cancer. Breast Cancer Res Treat 2010;121:121131. 
50 Taylor SK, Chia S, Dent S, et al.: A phase II study of pazopanib in patients with recurrent or metastatic invasive breast carcinoma: A trial of the Princess Margaret Hospital phase II consortium. Oncologist 2010;15: 810-818.

51 Robert NJ, Saleh MN, Paul D, et al.: Sunitinib plus paclitaxel versus bevacizumab plus paclitaxel for first-line treatment of patients with advanced breast cancer: A phase III, randomized, open-label trial. Clin Breast Cancer 2011;11:82-92.

52 Bergh J, Bondarenko IM, Lichinitser MR, et al.: Firstline treatment of advanced breast cancer with sunitinib in combination with docetaxel versus docetaxel alone: Results of a prospective, randomized phase III study. J Clin Oncol 2012;30:921-929.

53 Crown JP, Dieras V, Staroslawska E, et al.: Phase III trial of sunitinib in combination with capecitabine versus capecitabine monotherapy for the treatment of patients with pretreated metastatic breast cancer. J Clin Oncol 2013;31:2870-2878.

54 Schwartzberg LS, Tauer KW, Hermann RC, et al. Sorafenib or placebo with either gemcitabine or capecitabine in patients with HER-2-negative advanced breast cancer that progressed during or after bevacizumab. Clin Cancer Res 2013;19:2745-2754

55 Nielsen TO, Hsu FD, Jensen K, et al.: Immunohistochemical and clinical characterization of the basal-like subtype of invasive breast carcinoma. Clin Cancer Res 2004;10:5367-5374.

56 Rakha EA, El-Sayed ME, Green AR, et al.: Prognostic markers in triple-negative breast cancer. Cancer 2007; 109:25-32.
57 Di Leo A, Gomez HL, Aziz Z, et al.: Phase III, doubleblind, randomized study comparing lapatinib plus paclitaxel with placebo plus paclitaxel as first-line treatment for metastatic breast cancer. J Clin Oncol 2008; 26:5544-5552.

58 Finn RS, Press MF, Dering J, et al.: Estrogen receptor, progesterone receptor, human epidermal growth factor receptor 2 (HER2), and epidermal growth factor receptor expression and benefit from lapatinib in a randomized trial of paclitaxel with lapatinib or placebo as first-line treatment in HER2-negative or unknown metastatic breast cancer. J Clin Oncol 2009;27:3908-3915.

59 Balko JM, Cook RS, Vaught DB, et al.: Profiling of residual breast cancers after neoadjuvant chemotherapy identifies DUSP4 deficiency as a mechanism of drug resistance. Nat Med 2012;18:1052-1059.

60 O'Shaughnessy J, Weckstein DJ, Vukelja SJ, et al.: Preliminary results of a randomized phase II study of weekly irinotecan/carboplatin with or without cetuximab in patients with metastatic breast cancer. Breast Cancer Res Treat 2007;106 Suppl 1:S32 abstr 308.

61 Brown SD, Warren RL, Gibb EA, et al.: Neo-antigens predicted by tumor genome meta-analysis correlate with increased patient survival. Genome Res 2014;24: 743-750.

62 Nanda R, Chow LQ, Dees EC, et al.: Abstract S1-09: A phase Ib study of pembrolizumab (MK-3475) in patients with advanced triple-negative breast cancer. Cancer Res 2015;75:S1-09.

63 Emens LA, Braiteh FS, Cassier P, et al.: Abstract PD16: Inhibition of PD-L1 by MPDL3280A leads to clinical activity in patients with metastatic triple-negative breast cancer. Cancer Res 2015;75:PD1-6.

64 Yeatman TJ: A renaissance for Src. Nature Rev Cancer 2004;4:470-480.
65 Finn RS, Dering J, Ginther C, et al.: Dasatinib, an orally active small molecule inhibitor of both the src and abl kinases, selectively inhibits growth of basaltype/'triple-negative' breast cancer cell lines growing in vitro. Breast Cancer Res Treat 2007;105:319-326.

66 Huang F, Reeves K, Han X, et al.: Identification of candidate molecular markers predicting sensitivity in solid tumors to dasatinib: Rationale for patient selection. Cancer Res 2007;67:2226-2238.

67 Mayer E, Baurain J, Sparano J, et al.: Dasatinib in advanced HER2/neu amplified and ER/PR-positive breast cancer: Phase II study CA180088. J Clin Oncol 2009;27 Supp:15s abstr 1011

68 Marty B, Maire V, Gravier E, et al.: Frequent PTEN genomic alterations and activated phosphatidylinositol 3-kinase pathway in basal-like breast cancer cells. Breast Cancer Res 2008;10:R101.

69 Ueng SH, Chen SC, Chang YS, et al.: Phosphorylated mTOR expression correlates with poor outcome in early-stage triple negative breast carcinomas. Int J Clin Exp Pathol 2012;5:806-813.

70 Walsh S, Flanagan L, Quinn C, et al.: mTOR in breast cancer: Differential expression in triple-negative and non-triple-negative tumors. Breast 2012;21:178-182.

71 Huober J, Fasching PA, Hanusch C, et al.: Neoadjuvan chemotherapy with paclitaxel and everolimus in breast cancer patients with non-responsive tumours to epirubicin/cyclophosphamide (EC) +/- bevacizumab - Results of the randomised geparquinto study (GBG 44). Eur J Cancer 2013;49:2284-2293.

72 Balko JM, Giltnane JM, Wang K, et al.: Molecular profiling of the residual disease of triple-negative breast cancers after neoadjuvant chemotherapy identifies actionable therapeutic targets. Cancer Discov 2014; 232-245. 